\title{
Infracción tributaria y utilidad: un análisis correlacional en empresas del emporio Gamarra
}

\author{
Tax violation and utility: a correlational analysis in companies from the Gamarra \\ emporium
}

\author{
Jane Lucia Sihuacuyo Rodriguez ${ }^{1^{*}}$, Luz Marina Arisaca Llanque ${ }^{1}$ \\ ${ }^{1}$ Escuela Profesional Contabilidad, Facultad de Ciencias Empresariales, Universidad Peruana Unión
}

INFORMACIÓN DEL ARTÍCULO

Historia del artículo

Recibido: 27 setiembre de 2017

Aceptado: 20 enero de 2018
Palabras clave:

Infracción tributaria, utilidad.

\section{Resumen}

El nivel de carga tributaria promedio en América Latina esconde una marcada heterogeneidad de casos en el aspecto tributario, siendo el incumplimiento, cumplimiento parcial, cumplimiento incorrecto o cumplimiento tardío de las obligaciones tributarias lo que acarrea en infracciones tributarias, que muchos emprendedores peruanos se hacen acreedores; de allí que, este estudio determina la relación que existe entre infracciones tributarias y la utilidad de las empresas del emporio de Gamarra, durante los periodos 2012-2015 Lima Perú. El tipo de estudio es descriptivo-correlacional, de diseño no experimental, se analizó la información emanada de las declaraciones, notificaciones o esquelas de la SUNAT y estados financieros de una muestra de 20 empresas textiles. Los resultados determinan una relación significativa entre las infracciones relacionadas con la obligación de llevar libros y/o registros o contar con informes u otros documentos, con la utilidad neta $(r=-0,232 ; p$ valor $=0,038<0.05)$. Por tanto, el estudio brinda un análisis de las clases de infracciones más relevantes en todas las empresas, para el cuidado de sus operaciones, sin afectar su utilidad.

\footnotetext{
Abstrac

The The average level of tax burden in Latin America hides a marked heterogeneity of cases in the tax aspect, including noncompliance, partial compliance, incorrect compliance or late compliance with tax obligations that leads to tax violations, which many Peruvian entrepreneurs commit; hence, this study determines the relationship that exists between tax violations and the utility of the companies in the Gamarra emporium, during the periods 20122015 in Lima, Peru. The type of study is descriptive-correlational, of a non-experimental design, using the information from SUNAT declarations, notifications or obituaries and financial statements of a sample of 20 textile companies. The results show a significant relationship between violations related to the obligation to keep books and/or records or to have reports or other documents, with net profit $(r=-0.232 ; p$ value $=0.038<0.05)$. Therefore, the study provides an analysis of the most relevant types of infringements in all companies, for the care of their operations, without affecting their bottom line.
}

Keywords:

Tax infringement, utility 


\section{Introducción}

De acuerdo con cifras de la Comisión Económica para América Latina y el Caribe (CEPAL) y la Superintendencia $\mathrm{Na}$ cional de Aduanas y Administración Tributaria (SUNAT), Moran y Pecho (2016) el Perú es uno de los países con un alto grado de evasión tributaria, alcanzando un 34,7\% (Quintanilla, 2014).

El nivel de carga tributaria promedio en América Latina esconde una marcada heterogeneidad de casos, tanto en el aspecto tributario, como en el aspecto social y económico; así en principio, puede observarse que Brasil, Bolivia y Argentina son los países con mayor carga tributaria en el sub-período más reciente (entre $23,4 \%$ y $25,9 \%$ para $2010-2014$ ).

Es así como, en el Perú existe una elevada tasa de evasión, morosidad entre otros; es así que la estimación de incumplimiento del impuesto general a las ventas desde el año 2003 al 2014 fue: 46,3\% en el $2003 ; 45,5 \%$ en el $2004,43,1 \%$ en el $2005 ; 38,9 \%$ en el $2006 ; 36,2 \%$ en el $2007 ; 30,8 \%$ en el $2008,38,7 \%$ en el $2009 ; 32,7 \%$ en el $2010 ; 29,3 \%$ en el $2011 ; 29,2 \%$ en el $2012 ; 28,3 \%$ en el 2013 y $28,3 \%$ en el 2014 (SUNAT, 2015).

De acuerdo con el estudio de Manrique (2015), en el callao, el $62.50 \%$ de los consumidores finales contribuyen a que el cumplimiento de las obligaciones tributarias sea baja, el $29.17 \%$ determinan que sea medio y solo el $8.33 \%$ determinan a que el cumplimiento sea alto. Por su parte, Choquecahua (2016), en un estudio realizado en el emporio de Gamarra, señala que un $22 \%$ no entregan comprobantes de pago, un $78 \%$ no respetan sus obligaciones formales, un $50 \%$ no cumple con su obligación tributaria y un $61 \%$ no cumplen con sus obligaciones sustanciales. Como consecuencia, los contribuyentes que evaden sus obligaciones se convierten en infractores, afectando su economía por las sanciones recibidas.

Por su parte Burga (2015), afirma que en los negocios existen comerciantes con poca conciencia tributaria, asimismo el emporio de Gamarra no es ajeno a este problema, ya que existe un comportamiento inadecuado de los contribuyentes, esto se debe a falta de información, conocimiento y capacitación en el ámbito tributario. Es así que el sector textil de gamarra viene experimentando un crecimiento acelerado; en la actualidad, aproximadamente el $80 \%$ de artículos textiles es realizado a través de este mercado de Gamarra, sin embargo, la evasión tributaria es elevada y muchos talleres que destinan su producción a Gamarra no pagan impuestos generando sanciones (Gomero, 2003).

Ahora bien, uno de los problemas que afectan tanto a Gamarra como a otros centros comerciales, es el uso de facturas falsas, en las cuales el contribuyente busca evadir, confeccionando, obteniendo, vendiendo o crédito o notas de débito, con el objeto de cometer o posibilitar delitos tipificados en la ley penal tributaria, como son la defraudación tributaria y el delito tributario contable (Yacolca, 2012).

Sin duda alguna, debido a que diversas empresas se han visto afectadas por infracciones tributarias, que causan malestar al contribuyente, se han realizado investigaciones como el de (Burga, 2015b), quien, en los resultados de su investigación, encuentra que existe una relación significativa entre concientización tributaria y cumplimiento de las obligaciones tributarias, es decir, que a mayor concientización tributaria, mayor cumplimiento habrá , o lo contrario mientras menos concientización exista, menos cumplimiento existirá. Por su parte, Choquecahua(2016), afirma y encuentra 
que el conocimiento tributario incide significativamente en el cumplimiento de obligaciones tributarias.

A su vez, Calderon y Jauregui (2016), determinan que existe una relación altamente significativa entre control interno y cumplimiento de obligaciones tributarias, es decir, que, manteniendo un buen nivel de control interno, menores serán los riesgos tributarios referentes a sanciones y multas. De manera similar Galicia (2016), atribuye que el nivel de incumplimiento de las obligaciones tributarias está relacionado en mayor porcentaje a no pagar los tributos dentro de los plazos establecidos, y que un bajo nivel de cultura obstaculiza el cumplimiento de obligaciones tributarias.

En tanto que, Granda y Noboa (2015), establecen que el no cumplimiento de las obligaciones tributarias disminuye la utilidad de las empresas. Siendo necesario realizar un control permanente de los estados contables financieros de la empresa con el objeto de garantizar una información veraz de acuerdo a la realidad económica de la empresa sin que esto afecte a la utilidad. (Dueñas, 2016)

Ante las afirmaciones consideradas en los párrafos que anteceden, el presente estudio determina si efectivamente las infracciones tributarias están relacionadas con la utilidad de las empresas del emporio de Gamarra.

\section{Revisión de la literatura}

\section{Infracción Tributaria}

En palabras de Guerra (2015), la infracción se determina de manera objetiva, no requiere intencionalidad $\mathrm{y}$, mayormente, está relacionada al incumplimiento de obligaciones formales; pudiendo ser de orden formal y sustancial. El orden formal está relacionado con la presentación de las declaraciones en los plazos previstos por la administración tales como: emisión de comprobantes de pago, obligación de llevar libros contables e inscripción en el registro único del contribuyente (RUC); determinación y declaración de los tributos en los plazos establecidos legalmente, sustentación con guías de remisión cuando se realice traslado de bienes, entre otros (Burgos y Gutierrez, 2013). En tanto que, las obligaciones de orden sustancial, son aquellas vinculadas a la obligación de no omitir ingresos (Arias, 2005), como pagar oportunamente los tributos tales como: impuesto a la renta, impuesto general a las ventas, pagar las retenciones y percepciones, depositar detracciones de acuerdo con las normas vigentes(Burgos y Gutierrez, 2013).

Según Vera y Basallo (2004), y en concordancia con el artículo 166 del Código Tributario, manifiesta que toda infracción tributaria es sancionada por la administración tributaria y es ejercida de modo discrecional. Al respecto, Bahamonde (2014), señala que, toda infracción se sanciona con penas pecuniarias, comiso de bienes, internamiento temporal del vehículo, cierre temporal de establecimiento $u$ oficina de profesionales independientes, entre otros más. De igual forma, (Arancibia, 2008), sostiene que la facultad sancionadora se manifiesta de modo discrecional y acota que dichas sanciones se pueden aplicar gradualmente. La facultad de graduar sanciones consiste en regular la aplicación de sanciones sobre la base de diversos tramos o escalas de acuerdo con el código tributario, además de utilizar algunos criterios como: frecuencia de la comisión de infracción, subsanación voluntaria o inducida del infractor, requisitos incumplidos entre otros más (Brun, 2004). 


\section{Utilidad}

Según Villanueva (2004), la utilidad es un beneficio empresarial, como una ganancia en un determinado periodo en la medida en que se produzca un aumento en la riqueza (Skiarski, 2013). En tal sentido, es la cantidad de riqueza que aunque sea utilizado no afecta el capital de la empresa, debido a que es un excedente del patrimonio inicial (Uribe, 2006; citado en Alvarez y Contreras, 2015). Convirtiéndose en el mejor indicador de desempeño. (Dechow, 1994; citado por Jara y López, 2011).

Por otro lado, la utilidad proviene de la diferencia entre lo que una empresa invierte en sus costos para fabricar bienes o realizar servicios y los ingresos que se obtienen por venderlos (Naranjo y Naranjo, 2005; citado en Toapanta, 2014), mostrado como un resultado expresado en forma positiva reflejo de la situación financiera (Delgado, 1988). En suma, una empresa ha obtenido utilidad cuando luego de haber deducido los gastos de los ingresos queda un saldo conocido también como remanente (Rengifo y Vigo, 2014).

\section{Materiales y métodos}

El presente estudio es de tipo descriptivo correlacional, con un diseño no experimental, bajo la determinación de muestreo por conveniencia se tuvo como muestra 20 empresas textiles. Además, para la recolección y tratamiento de datos y para la obtención de la utilidad se tomaron en cuenta la sistematización de información de los estados financieros considerando: utilidad bruta, utilidad operativa y utilidad neta. Para el análisis de infracción tributaria se obtuvo información sistematizada de las notificaciones y/o esquelas de SUNAT, priorizando las infracciones más relevantes: Infracciones relacionadas con la obligación de llevar libros y/o registros, infracciones relacionadas con la obligación de presentar declaraciones de naturaleza determinativa o informativa e infracciones relacionadas con el cumplimiento de las obligaciones tributarias: omisión de ingresos, declaración de cifras o datos falsos, no pagar los tributos retenidos o percibidos dentro de los plazos establecidos.

\section{Resultados}

\section{Resultados descriptivos}

Según el análisis comparativo de la utilidad de las empresas del emporio de Gamarra durante los periodos 2012-2015, en la tabla 4 se observa que el promedio de la utilidad bruta es de 0.24 , con una utilidad mínima de 0.08 y la máxima de 0.57 y una desviación estándar de 0.13 , mientras que en la utilidad operativa el promedio es de 0.10 , con una utilidad mínima de 0.02 y la máxima de 0.23 y una desviación estándar de 0.06 y finalmente en la utilidad neta el promedio es de 0.05 con una utilidad mínima de 0.00 y la máxima de 0.18 y una desviación estándar de 0.03 , es decir, el promedio de utilidad bruta es mayor que el de utilidad operativa y neta.

Tabla 1

Utilidad de las empresas del emporio de Gamarra, periodo 2012-2015

\begin{tabular}{lcccc} 
& $M$ & $D S$ & Mín. & Máx. \\
& & & & \\
\hline Utilidad Bruta & 0.24 & 0.13 & 0.08 & 0.57 \\
Utilidad Operativa & 0.10 & 0.06 & 0.02 & 0.23 \\
Utilidad Neta & 0.05 & 0.03 & 0.00 & 0.18
\end{tabular}

En la tabla 2, se observa el promedio de la utilidad bruta, determinándose que el $65 \%$ de las empresas, se encuentran por debajo del promedio, mientras que el $35 \%$, está por encima del promedio de la 
utilidad. En la utilidad operativa se observa que el $50 \%$ de las empresas, se encuentra por debajo del promedio al igual que el $50 \%$, está por encima del promedio de la utilidad. En la utilidad neta se observa que el $50 \%$ está por encima del pro- medio de la utilidad al igual que el 50\% está por encima del promedio de la utilidad; es decir, se observa que utilidad bruta se encuentra por debajo del promedio, mientras que la utilidad operativa y neta está por encima del promedio.

Tabla 2

Promedio de la utilidad en las empresas del emporio de Gamarra, periodo 2012-2015

\begin{tabular}{|c|c|c|c|c|c|c|}
\hline \multirow[b]{2}{*}{$\begin{array}{l}\text { Utilidad Bruta } \\
\text { (promedio } \\
23 \%)\end{array}$} & \multicolumn{2}{|c|}{$\begin{array}{l}\text { Por debajo del prome- } \\
\text { dio de la utilidad }\end{array}$} & \multicolumn{2}{|c|}{$\begin{array}{c}\text { Por debajo del promedio de la } \\
\text { utilidad }\end{array}$} & \multicolumn{2}{|c|}{ Total } \\
\hline & 52 & $65.0 \%$ & 28 & $35.0 \%$ & 80 & $100 \%$ \\
\hline $\begin{array}{l}\text { Utilidad Opera- } \\
\text { tiva } \\
\text { (promedio }=9 \% \text { ) }\end{array}$ & 40 & $50.0 \%$ & 40 & $50.0 \%$ & 80 & $100 \%$ \\
\hline $\begin{array}{l}\text { Utilidad Neta } \\
\text { (promedio }=5 \% \text { ) }\end{array}$ & 40 & $50.0 \%$ & 40 & $50.0 \%$ & 80 & $100 \%$ \\
\hline
\end{tabular}

En la tabla 3, se observa la cantidad total de infracciones que han incumplido las empresas del emporio de Gamarra durante los periodos 2012-2015. En las infracciones tributarias el $66.3 \%$ de las empresas presenta una infracción, mientras que el $23.8 \%$ presenta dos infracciones, el $6.3 \%$ presenta tres infracciones y finalmente el $3.8 \%$ no presenta ninguna infracción, es decir, que el mayor porcentaje de empresas presenta al menos una infracción.

Tabla 3

Cantidad de infracciones tributarias de las empresas del emporio Gamarra, periodo 2012-2015

\begin{tabular}{lcc}
\hline Infracciones tributarias & $n$ & $\%$ \\
\hline Sin infracción & 3 & 3.8 \\
Con 1 infracción & 53 & 66.3 \\
Con 2 infracciones & 19 & 23.8 \\
Con 3 infracciones & 5 & 6.3 \\
\hline Total & 80 & 100.0
\end{tabular}

\section{Resultados inferenciales}

En la tabla 4 se presenta la correlación entre los indicadores de infracciones relacionadas con la obligación de llevar libros y/o registros o contar con informes $u$ otros documentos y la utilidad neta de las empresas del emporio de gamarra durante los periodos 2012-2015. Mediante el análisis estadístico Rho de Spearman, se obtuvo el coeficiente de $-0,232^{*}$ y una significancia de un $p$ valor de 0,038 ( $p$ valor $<$ 0,05 ); por lo tanto, las infracciones relacionadas con la obligación de llevar libros y/o registros o contar con informes $u$ otros documentos, se relaciona significativamente y directamente con la utilidad neta; por tanto, a mayor infracción menor utilidad.

Tabla 4

Relación entre utilidad netae infracciones relacionadas con la obligación de llevar libros y/o registros o contar con informes $u$ otros documentos 
Infracciones relacionadas con la obligación de llevar libros y/o registros o contar con informes $u$ otros documentos

\begin{tabular}{llll}
\hline & Rho de Spearman & $p$ valor & $n$ \\
\hline $\begin{array}{l}\text { Utilidad } \\
\text { neta }\end{array}$ &,$- 232^{*}$ & 0,038 & 80 \\
\hline
\end{tabular}

En la tabla 5, se muestra la relación entre infracciones relacionadas con el cumplimiento de las obligaciones tributarias y utilidad neta de las empresas del emporio de Gamarra durante los periodos 20122015. Asimismo, mediante el análisis estadístico Rho de Spearman se obtuvo el coeficiente de $-0,080$ y una significancia de un $p$ valor de 0,481 ( $p$ valor $>0.05$ ); por lo tanto, las infracciones relacionadas con el cumplimiento de las obligaciones tributarias no se relacionan significativamente con la utilidad neta; es decir no producen cambios significativos en la utilidad si existe cumplimiento con las obligaciones tributarias.

Tabla 5

Relación entre Infracciones relacionadas con el cumplimiento de las obligaciones tributarias y utilidad neta

\begin{tabular}{llll}
\hline & $\begin{array}{l}\text { Infracciones relacionadas con } \\
\text { el cumplimiento de las obliga- } \\
\text { ciones tributarias }\end{array}$ & \\
\hline & $\begin{array}{l}\text { Rho de Spear- } \\
\text { man }\end{array}$ & $\mathrm{p}$ valor & $\mathrm{n}$ \\
\hline $\begin{array}{l}\text { Utilidad } \\
\text { neta }\end{array}$ & -0.080 & 0.481 & 80 \\
\hline
\end{tabular}

\section{Discusión}

Según los resultados, la dimensión infracciones tributarias relacionadas a libros y/o registros y utilidad neta presentó una relación negativa inversa estadísticamente significativa con un coeficiente de $0.232^{*}$ y un $p$ valor de 0.038 ( $p$ valor < 0.05 ), es decir, mientras mayor sea la cantidad de veces que se cometa infracciones referentes a legalización de libros, uso indebido de comprobantes en las anotaciones de los libros y/o registros y no registrar todos los ingresos en los libros contables, menor será la utilidad neta. Al respecto Calvo (2009), considera que la infracción es considerada un medio de fraude, manifestándose en una relación causa-efecto; tal como la infracción por no realizar anotaciones de los ingresos en los libros de contabilidad. Por su parte, Nima et al. (2013), afirma que el sustento en las anotaciones efectuadas en los libros contables u otros libros, con comprobantes de pago $\mathrm{u}$ otros documentos falsificados $\mathrm{o}$ que no revelen los hechos que se pretenden sustentar, son sancionados; por tanto, toda infracción de una u otra manera repercute significativamente en sanciones económicas que pueden afectar la utilidad neta de una empresa.

Ahora bien, tomando en cuenta que las infracciones relacionadas al cumplimiento de obligaciones tributarias (Oyola, 2014), no producen cambios significativos en la utilidad neta (Alpaca, 2012), según los resultados del estudio al no encontrarse una relación estadísticamente significativa. No obstante, Granda y Noboa (2015), al analizar su variable cumplimiento de obligaciones tributarias encontraron una correlación negativa inversa altamente significativa, con un coeficiente de $-0,821^{\text {** }}$ y un $p$ valor de 0,001, es decir, que el pago de las sanciones fue elevado, lo que disminuyó la utilidad de la empresa, explicando que de no haberse producido retrasos en el cumplimiento de las obligaciones tributarias, no se habrían generado sanciones y éstas no hubieran afectado la utilidad de la empresa.

Por otro lado, Calderon y Jauregui (2016), al analizar el control interno y su relación con el cumplimiento de obligaciones tributarias; encontraron una relación 
altamente significativa, con un coeficiente de $0,600^{* *}(R$ Spearman) y un $p$ valor de $p$ $=0,008(p<0,005)$. Es decir, que el cumplimiento de obligaciones tributarias tiene mayor relación con la variable control interno, lo que evidencia que una empresa con controles internos bien establecidos tiene menos probabilidades de incumplir tributariamente y evitar posteriormente infracciones.

Finalmente, Galicia (2016), encontró un resultado similar al analizar cultura tributaria y su relación con el incumplimiento de las obligaciones tributarias, donde hallo un coeficiente de 0,691(Rho de Spearman) y un $p$ valor de 0,004 ( $p<0,005)$; mostrando una relación directa entre ambas variables, es decir, a mayor desconocimiento de cultura tributaria mayor es el incumplimiento y mayor aun serán las infracciones. Pese a ello, el hecho de que las empresas de gamarra incumplan con sus obligaciones tributarias, estas no están relacionadas directamente con la utilidad neta, por otros factores intervinientes.

\section{Conclusión}

El resultado de esta investigación sirve como medida preventiva, debido a que la relación existente entre las infracciones relacionadas a libros y/o registros, con la utilidad neta, implica que a mayor infracción en el cumplimiento de obligaciones formales y sustanciales, estarán sujetas a sanciones económicas y por ende repercutirá en obtener una menor utilidad. Siendo un tema relevante para las empresas, puesto que se encontró que entre las infracciones más recurrentes se destacan la omisión en la presentación de declaraciones, seguida por el cumplimiento de obligaciones tributarias y finalmente la obligación de llevar libros y/o registros. 


\section{Referencias}

Alpaca, A. (2012). El Uso de Costos Relevantes para la Toma de Decisiones Gerenciales y su Influencia en la Utilidad de las Empresas Industriales de la Ciudad de Tacna, año 2012. Universidad Nacional Jorge Basadre GrohmannTacna.

Alvarez, Ambar y Contreras, J. (2015). La Nic 18 en las ventas diferidas de vehículos y su influencia en la utilidad de la empresa Mannucci Diesel S.A.C. 2015. Universidad Privada Antenor - Trujillo.

Arancibia, M. (2008). Codigo Tributario Comentado y Concordado Jurisprudencia Sectorial. (Instituto Pacífico SAC, Ed.) (1st ed.). Lima.

Arias Scipion, C. (2005). Infracciones y Sanciones Tributarias: Facultad Discrecional de la Sunat. Revista Actualidad Empresarial, 91, 1-2.

Bahamonde Quinteros, M. (2014). ¿Como se determinan las Multas calculadas en función a los ingresos netos? Revista Caballero Bustamante, 791, 1-6.

Brun Herbozo, H. (2004). Comentarios al Nuevo Regimen de Incentivos y al Regimen de Gradualidad. Revista Actualidad Empresarial, 62, 1-4.

Burga Argandoña, M. E. (2015a). Cultura tributaria y Obligaciones tributarias en las empresas comerciales del emporio de Gamarra. Universidad de san martin de porres.

Burga Argandoña, M. E. (2015b). Cultura tributaria y Obligaciones tributarias en las empresas comerciales del emporio Gamarra, 2014. Universidad de San Martin de Porres.

Burgos Cruz, A. S., \& Gutierrez García, D. V. (2013). La Auditoria Tributaria Preventiva y su Impacto en el Riesgo Tributario en la empresa Inversiones Padre Eterno EIRL Año 2012.
Universidad Privada Antenor Orrego.

Calderon, Aida y Jauregui, K. (2016). El Control Interno y su Relacion con el Cumplimiento de las Obligaciones Tributarias en las empresas comerciales, Chaclacayo, 2015. Universidad Peruana Union.

Calvo, J. (2009). El papel de las anomalias sustanciales en contabilidad y en los libros o registros dentro de la calificación de la infracción tributaria. Cronica Tributaria, Numero 131, 7-34.

Choquecahua Ataucusi, S. (2016). Cultura tributaria y su incidencia en el cumplimiento de obligaciones tributarias de los empresarios de mypes en el emporio comercial gamarra, periodo 2015. Universidad Nacional del Callao.

Delgado, J. (1988). Algunas consideraciones acerca de la utilidad y el dividendo en las sociedades anónimas. Revista de Derecho Themis, 10, 38-41.

Dueñas, Y. (2016). Determinacion de la Utilidad Neta de la Empresa \&quot;JUAN ELJURI S.A en el ejercicio economico 2015, 15 .

Galicia, Y. (2016). La cultura Tributaria y su relación con el Incumplimiento de las obligaciones tributarias en las empresas del sector ferreterías del pueblo de Virú, Distrito den Virú, año 2015. Universidad César Vallejo.

Gomero Gonzales, A. N. (2003). Participacion de los intermediarios financieros bancarios y no bancarios en el desarrollo de las pymes textiles en lima metropolitana de 1990 al 2000. Universidad nacional mayor de san marcos.

Granda, Maribel y Noboa, rosa. (2015). Incidencia de las Obligaciones Tributarias en las Utilidades del comercial \&quot;Su Hacienda\&quot; del cantón general Antonio Elizalde (Bucay) 
del Año 2014. Universidad Estatal de Milagro - Ecuador.

Guerra Salvatierra, M. del pilar. (2015). Principales infracciones tributarias. Revista Actualidad Empresarial, Casos Prácticos (Parte I), 11-14.

Jara, Mauricio y López, F. (2011). La calidad e importancia de las utilidades contables para las empresas cotizadas en los mercados de capitales chilenos. Trimestre Economico, 643-674.

Manrique Ramos, M. A. (2015). Cultura tributaria del consumidor final y cumplimiento de las obligaciones tributarias del contribuyente de ventanilla. Universidad Nacional del Callao.

Moran, Eduardo y Pecho, M. (2016). La tributación en América Latina en los últimos 50 años, 6 .

Nima Nima, Elizabeth \& Rey Rojas, J. (2013). Aplicacion Práctica del Régimen de Infracciones y Sanciones Tributarias. Revista Contadores \& Empresas, 266.

Oyola, C. P. (2014). Infracciones Tributarias vinculadas al pago de los tributos retenidos, el caso del numeral 4 del artículo $178^{\circ}$ del Código Tributario. Revista Actualidad Empresarial, 22-23.

Quintanilla, E. (2014). La Evasión Tributaria y su incidencia en la recaudación Fiscal en el Perú y Latinoamérica. Universidad de San Martin de Porres.
Rengifo, Christian y Vigo, A. (2014). Incidencia de las Infracciones Tributarias en la situación Económica y Financiera de las mypes del sector calzado Apiat - 2013. Universidad Privada Antenor Orrego Trujillo.

Skiarski, E. M. (2013). Más sobre el concepto de ganancias realizadas y líquidas. In VII Congreso Iberoamericano de Derecho Societario y de la Empresa (pp. 451454). Buenos Aires. Retrieved from https://repositorio.uade.edu.ar/xmlui/bits tream/handle/123456789/436/CDS1203 0451. pdf?sequence $=1$

Superintendencia Nacional de Aduanas y Administracion Tributaria. (2015). Informe de Gestión por Resultados 2015 SUNAT, 11.

Toapanta, D. (2014). La Contabilidad de Gestión y su Incidencia en la Utilidad Contable de los productos elaborados por Industrias y Textiles Pequeñin Cía. Ltda., en el primer trimestre de año 2013. Universidad Técnica de Ambato Ecuador.

Vera, Isaías y Basallo, C. (2004). Comentarios al Codigo Tributario. (Editores e Impresiones Surco S.A., Ed.) (2nd ed.). Lima.

Villanueva González, M. (2004). Las Rentas Empresariales. Jornadas Nacionales De Derecho Tributario, 1-13.

Yacolca Estares, D. (2012). Nuevo delito Tributario de facturas falsas. Revista Actualidad Empresarial, 2. 